\title{
T形腔熕耗散最小构形优化
}

\author{
谢志辉，陈林根 ${ }^{*}$, 孙丰瑞 \\ 海军工程大学研究生院, 武汉 430033 \\ * 联系人, E-mail: lingenchen@hotmail.com, 1gchenna@yahoo.com \\ 2009-03-23 收稿, 2009-05-05 接受
}

教育部新世纪优秀人才支持计划(编号: NCET-04-1006)和全国优秀博士学位论文作者专项资金(编号: 200136)资助项目

摘要 积耗散极值原理给出了新的传热优化的理论依据和评判标准. 针对矩形固体中包含 $\mathrm{T}$ 形开口空腔的传热模型, 引入了基于炽耗散定义的无量纲当量热阻, 在系统总体积、空腔体 积以及 $\mathrm{T}$ 形腔所占矩形域体积一定的约束条件下, 以该当量热阻最小化为目标对模型进行 了构形优化. 数值结果表明, 基于炇耗散极值原理可以设计出系统传热性能最优的几何结 构. 修正了文献中无量纲总(最大)热阻的表达式, 以其最小化为目标得到了一些与文献结果 不同的新规律. 将两种热阻指标下的数值优化结果进行了对比分析, 发现分别对应两种指标

关键词

构形理论

炇耗散极值原理

传热

空腔

广义热力学优化 的最优系统结构明显不同, 以当量热阻最小化为目标的优化比以最大热阻最小化为目标的 优化, 可以有效降低传热平均温差, 改善系统整体传热性能. 空腔自由度越大, 系统性能更 佳. 通过数据拟合, 分别得到了当量热阻和最大热阻与 3 自由度几何参数的优化关联式.

固体中包含开口冷却空腔的传热模型普遍存在 于高热流密度和大热流量的现代工程领域中，随着 高性能化和小型化的进一步发展，对该类模型进行 优化设计以寻求最佳整体性能尤为重要. 优化传热 性能的理论依据主要有过增元等提出的场协同理论 $\stackrel{[1 \sim 3]}{ }$, Bejan等人 ${ }^{[4 \sim 6]}$ 提出的摘产最小原则和构形理论 [7 17]. 熵产最小原则是基于热、功转化过程中最大限 度输出功的思路, 以可用能损失最小为目标的优化 理论. 将熵产最小原则应用于传热和流体流动过程 特别是应用于换热器的优化设计时, 出现了悖论和 矛盾 ${ }^{[18,19]}$. 传热学研究的目的在于分析传热规律及 速率, 不一定要关注传热引起的可用能损失. 为了反 映传热的本质属性, 过增元等人 ${ }^{[20,21]}$ 基于经典的热 电比拟方法, 定义了表征物体热量传递总能力的新 物理量一一炇, 提出了积耗散极值原理 ${ }^{[20]}$, 引起了许 多学者的浓厚兴趣, 开展了以䛈耗散最小为目标的 传热优化研究 ${ }^{[22 ~ 32]}$, 并从导热机理 ${ }^{[27]}$ 、电热模拟试验
[28]等方面进一步阐述了积的物理意义, 在分别以摘 产极值和炇耗散极值为优化目标进行比较研究的基 础上, 指出各自适用对象不同, 对传热问题沵耗散极 值原理优于熵产最小原则 ${ }^{[24,25,31]}$. 魏曙寰等人 ${ }^{[33,34]}$ 将 炇耗散极值原理与构形理论结合起来进行研究, 夏 少军等人 ${ }^{[35]}$ 将积耗散极值原理与有限时间热力学理 论结合起来进行研究, 得到了传热性能更优的系统 构形和换热器构型.

构形理论认为随时间延续(或生存)的有限尺寸 流动系统, 它必定会循着为流经它的流提供更容易 的通道而不断进化 ${ }^{[7 ~ 17]}$. 无论是在自然界还是在工程 领域, 分叉都是普遍的事物进化(生成)模式, 在一定 约束下寻求整体性能最优的流道最终进化(优化)成

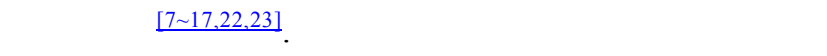
的 T形结构, 文献[8]以热流率最大为目标对 T形肋片 进行了构形优化, 文献[9]以流阻最小为目标分别对 T 形和Y形管道进行了构形优化, 文献[36]以无量纲最 
大温差最小为目标, 对矩形固体中包含的 $\mathrm{T}$ 形开口空 腔进行了构形优化. 本文在文献[36]的基础上, 以提 高整体传热性能为目的, 基于沵耗散极值原理对矩 形固体中T形开口空腔模型进行构形优化研究, 得到 系统构型与系统传热性能新的数值优化规律, 可为 相关热设计问题提供一定理论依据. 同时以传统的 最大热阻为目标函数采取同样方法进行优化研究, 分析比较两种优化结果的异同, 进一步深入研究系 统积耗散的规律.

\section{1 问题描述}

\section{1 熕耗散的定义}

文献[20]定义了一物体所具有的热量传递的总 能力——物理量炇 $\left(E_{V h}\right)$

$$
E_{V h}=Q_{V h} T / 2,
$$

其中 $Q_{V h}=M c_{V} T$ 为物体的定容热容量, $T$ 是物体温度. 由此得到了单位时间单位体积内积的耗散率, 称为积 耗散函数为 ${ }^{[20]}$ :

$$
\phi_{h}=-\dot{q} \cdot \nabla T=k(\nabla T)^{2},
$$

其中 $\dot{q}$ 为热流密度矢量, $\nabla T$ 是温度梯度.

整个体积中的炇耗散率为

$$
\dot{E}_{V h \phi}=\int_{V} \phi_{h} \mathrm{~d} V .
$$

在此基础之上，对于给定热流边界条件的多维 导热问题, 物体的当量热阻为 ${ }^{[20]}$

$$
R_{h}=\dot{E}_{V h \phi} / \dot{Q}_{h}^{2},
$$

其中 $\dot{Q}_{h}$ 是通过边界的热流率. 多维导热问题的平均 温差为 ${ }^{[20]}$

$$
\Delta \bar{T}=R_{h} \dot{Q}_{h}
$$

\section{2 传热模型}

考虑如图 1 所示的矩形导热固体 $(H \times L \times W), W$ 表示垂直纸面的第三维, 不考虑参数在 $W$ 方向的变 化, 模型简化为二维情形. 本文中的约束条件为系 统、空腔及 $\mathrm{T}$ 形腔所占矩形域的体积一定, 即

$$
\begin{gathered}
V=H L W=\text { Const }, \\
V_{c}=\left(2 L_{0} H_{0}+L_{1} H_{1}\right) W=\text { Const }, \\
V_{T}=2 L_{0}\left(H_{0}+L_{1}\right) W=\text { Const. }
\end{gathered}
$$

也可表达为总面积、空腔占比和 $\mathrm{T}$ 形腔所占矩形域占
比一定，即

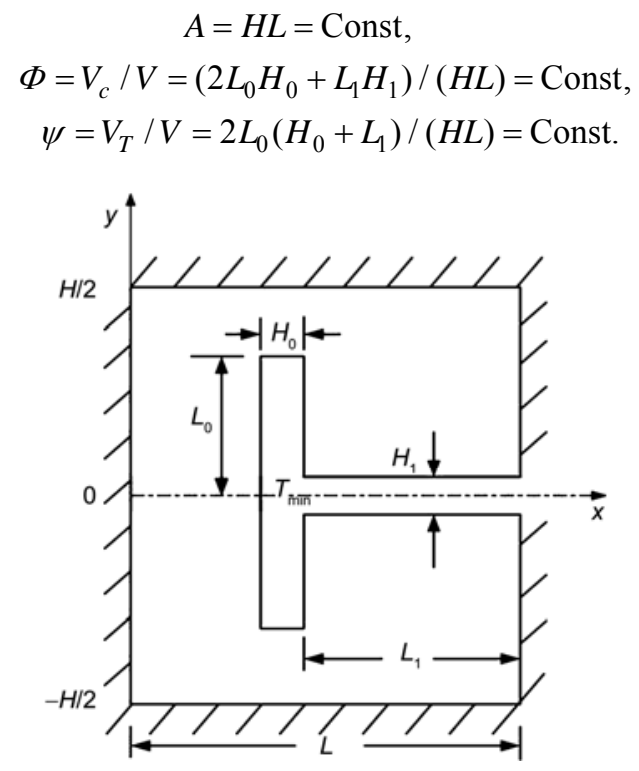

图 1 模型与边界

导热固体各相同性, 热导率 $k$ 为常数, 容积均匀 产热率为 $q^{\prime \prime \prime}\left[\mathrm{W} / \mathrm{m}^{3}\right]$, 外表面绝热. 空腔的主干 $\left(H_{1} \times L_{1}\right)$ 以垂直角度发展出两个对称分枝 $\left(H_{0} \times L_{0}\right)$, 生成T形空腔. 固体内部产生的热量传到低温的空腔 壁面后经对流换热传往外界. 为了重点突出优化方 法, 简化物理模型, 假设表面对流换热能力远远强于 固体的热传导能力, 即表面对流换热热阻远小于导 热热阻, 流体和空腔表面保持恒定的低温 $T_{\min }$, 忽略 流动的影响 ${ }^{[36]}$.

定义无量纲量为

$$
\begin{gathered}
\left(\tilde{x}, \tilde{y}, \widetilde{H}, \widetilde{L}, \widetilde{H_{0}}, \widetilde{L_{0}}, \widetilde{H_{1}}, \widetilde{L_{1}}\right)=\frac{\left(x, y, H, L, H_{0}, L_{0}, H_{1}, L_{1}\right)}{A^{1 / 2}}, \\
\widetilde{T}=\frac{T-T_{\min }}{\left[q^{\prime \prime \prime}(1-\Phi) A\right] / k} .
\end{gathered}
$$

几何约束可表达为

$\widetilde{H} \widetilde{L}=1, \quad \Phi=2 \widetilde{L}_{0} \widetilde{H}_{0}+\widetilde{L}_{1} \widetilde{H}_{1}, \quad \psi=2 \widetilde{L}_{0}\left(\widetilde{H}_{0}+\widetilde{L}_{1}\right)$.

无量纲导热方程为

$$
\frac{\partial^{2} \tilde{T}}{\partial \tilde{x}^{2}}+\frac{\partial^{2} \tilde{T}}{\partial \tilde{y}^{2}}+\frac{1}{1-\Phi}=0 .
$$

对应边界条件为：

固体外表面, $\partial \tilde{T} / \partial \tilde{n}=0$; 空腔表面, $\tilde{T}=0$.

根据(4)式, 当量热阻为 


$$
\begin{aligned}
R_{h} & =\frac{\int_{A} k(\nabla T)^{2} \mathrm{~d} \Omega}{\left[q^{\prime \prime \prime}(1-\Phi) A\right]^{2}}=\frac{\int_{H} \int_{L} k\left[(\partial T / \partial x)^{2}+(\partial T / \partial y)^{2}\right] \mathrm{d} x \mathrm{~d} y}{\left[q^{\prime \prime \prime}(1-\Phi) A\right]^{2}} \\
& =\frac{\int_{\widetilde{H}} \int_{\tilde{L}}\left[(\partial \tilde{T} / \partial \tilde{x})^{2}+(\partial \tilde{T} / \partial \tilde{y})^{2}\right] \mathrm{d} \tilde{x} \mathrm{~d} \tilde{y}}{k},
\end{aligned}
$$

所以无量纲当量热阻可定义为

$$
\widetilde{R}_{h}=R_{h} k=\int_{\widetilde{H}} \int_{\widetilde{L}}\left[(\partial \tilde{T} / \partial \tilde{x})^{2}+(\partial \tilde{T} / \partial \tilde{y})^{2}\right] \mathrm{d} \tilde{x} \mathrm{~d} \tilde{y} .
$$

根据(5)式, 多维导热问题的平均温差 $\Delta \bar{T}$ 为

$$
\begin{aligned}
\Delta \bar{T} & =R_{h} \dot{Q}_{h} \\
& =\frac{\int_{\widetilde{H}} \int_{\tilde{L}}\left[(\partial \tilde{T} / \partial \tilde{x})^{2}+(\partial \tilde{T} / \partial \tilde{y})^{2}\right] \mathrm{d} \tilde{x} \mathrm{~d} \tilde{y}}{k} q^{\prime \prime \prime}(1-\Phi) A .
\end{aligned}
$$

无量纲平均温差 $\widetilde{\Delta T}$ 为

$$
\begin{aligned}
\Delta \widetilde{\bar{T}} & =\frac{\Delta \bar{T}}{q^{\prime \prime \prime}(1-\Phi) A / k} \\
& =\int_{\widetilde{H}} \int_{\tilde{L}}\left[(\partial \widetilde{T} / \partial \tilde{x})^{2}+(\partial \tilde{T} / \partial \tilde{y})^{2}\right] \mathrm{d} \tilde{x} \mathrm{~d} \tilde{y}=\widetilde{R}_{h} .
\end{aligned}
$$

文献[36]采用的无量纲最大温差为

$$
\widetilde{T}_{\max }=\frac{T_{\max }-T_{\min }}{q^{\prime \prime \prime} A / k} .
$$

以(16)式最小化为目标进行构形优化对于防止 热点过热具有一定意义, 但文献[36]将其等同于无量 纲总(最大)热阻的概念是不准确的. 正确的无量纲最 大热阻应为

$$
\widetilde{R}_{t}=\frac{T_{\max }-T_{\min }}{\left[q^{\prime \prime \prime}(1-\Phi) A / k\right]} .
$$

(13)和(17)式虽然都与系统温度相关，但是两者 存在很大不同. (17)式是基于系统最大温差和实际散 热负荷定义的无量纲最大热阻. 由于最大温差是局 部意义的, 所以基于最大温差的无量纲热阻是以局 部极限特征刻画系统整体性能, 有一定的片面性和 局限性, 以其最小化为目标进行构形优化主要是出 于安全性考虑, 但物理上并不能表示系统的平均散 热性能最优. 而(13)式表明无量纲当量热阻是无量纲 温度梯度的模在整个区域的积分, 更能反映出“场” 的特征并刻画多维导热系统的平均散热性能，即当 量热阻越小, 传热效率越高, 结构体内的平均温差越 低, 传热性能越好 ${ }^{[34 \sim 36]}$.

\section{2 数值结果与讨论}

基于上述模型, 首先采用有限元法对图 1 所示模 型的不同几何结构求解出温度场, 然后计算得到当 量热阻和最大热阻的数值解, 再研究系统几何参数 与系统性能的优化关系. 由于模型具有对称性, 只需 计算 $y>0$ 部分. 计算精度控制为

$$
\left|\left(\widetilde{R}_{h}^{j}-\widetilde{R}_{h}^{j-1}\right) / \widetilde{R}_{h}^{j-1}\right|<0.005,
$$

式中 $(j-1)$ 表示对应前一次网格数的计算值, $j$ 则表示 对应加密 1 次后网格数的计算值. 若式(18)不满足, 则 加密一次网格, 重新计算, 直至达到精度要求为止. 为突出优化方法, 将模型简化为 $H / L=1$ 的情况. 首先, 在系统、空腔及 $\mathrm{T}$ 形腔所占矩形域体积一定的约束下, 给定 $H_{0} / H_{1}$, 通过最小化(13)式所示目标函数 $\widetilde{R}_{h}$ 或 (17)式所示目标函数 $\widetilde{R_{t}}$ 对 $L_{0} / L_{1}$ 进行一维优化, 然后 针对其他的 $H_{0} / H_{1}$ 进行同等精度的二维优化.

图 2 给出了一个针对 $L_{0} / L_{1}$ 的一维优化算例. 数 值结果表明，基于本文方法可以设计出对应目标函 数极值的系统构形, 对应 $\left(\widetilde{R_{h}}\right)_{\text {min }}$ 和 $\left(\widetilde{R_{t}}\right)_{\min }$ 的系统构 形明显不同, $\left(L_{0} / L_{1}\right)_{o p t}$ 分别为 0.39 和 $0.59,\left(\widetilde{R_{h}}\right)_{\min }$ 为 0.062478 , 而对应于 $\left(\widetilde{R_{t}}\right)_{\min }$ 的 $\widetilde{R_{h}}$ 为 0.071182 . 所 以对应前者的体内传热平均温差比后者的要低 $13.9 \%$, 因此以 $\left(\widetilde{R_{h}}\right)_{\text {min }}$ 为目标可以设计出系统内传热 平均温差最小的系统构形, 提高传热效率. 下文将在 给定系统体积的总约束下, 分别研究 $\mathrm{T}$ 形腔所占矩形 域占比 $\psi$ 一定时, 空腔占比 $\Phi$ 对系统构形及其性能 的影响; 空腔占比 $\Phi$ 一定时, $\mathrm{T}$ 形腔所占矩形域占比 $\psi$ 对系统构形及其性能的影响以及进一步释放 $\mathrm{T}$ 形腔 占矩形域占比 $\psi$ 的优化.

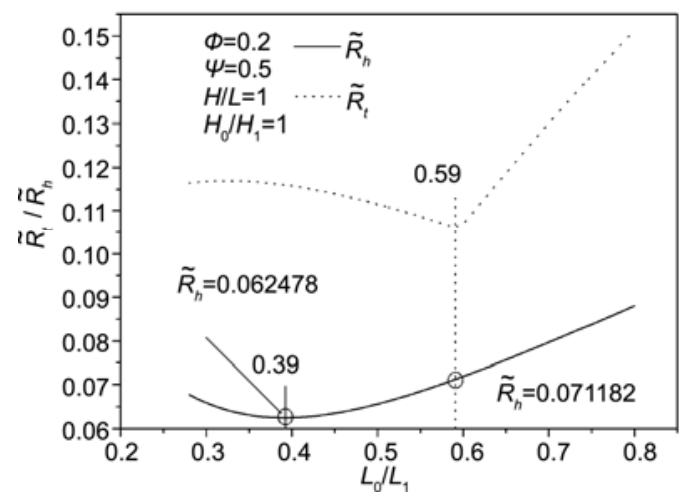

图 2 针对 $L_{0} / L_{1}$ 的一维优化 


\section{1 空腔占比 $\Phi$ 对系统构形及其性能的影响}

图 3 给出了分别以 $\left(\widetilde{R_{h}}\right)_{\text {min }}$ (实线)和 $\left(\widetilde{R_{t}}\right)_{\text {min }}$ (虚线) 为目标时 $\Phi$ 对 $\left(L_{0} / L_{1}\right)_{o p t}-H_{0} / H_{1}$ 关系的影响. 由图 可知, 当 $\Phi<0.5$ 时, 分别以 $\left(\widetilde{R_{h}}\right)_{\min }$ 和 $\left(\widetilde{R_{t}}\right)_{\min }$ 为目标 的优化结果区别很大, 但当 $\Phi=0.5$ 时, 由于尺寸的 限制, $\left(L_{0} / L_{1}\right)_{o p t}$ 都取到 $L_{0} / L_{1}$ 的最小值, 以 $\left(\widetilde{R_{h}}\right)_{\text {min }}$ 和 $\left(\widetilde{R_{t}}\right)_{\min }$ 为目标的优化构形相同. 此外, 不论采用哪 个指标作为优化目标, $\Phi$ 对 $\left(L_{0} / L_{1}\right)_{o p t}-H_{0} / H_{1}$ 关系 均具有较大影响.

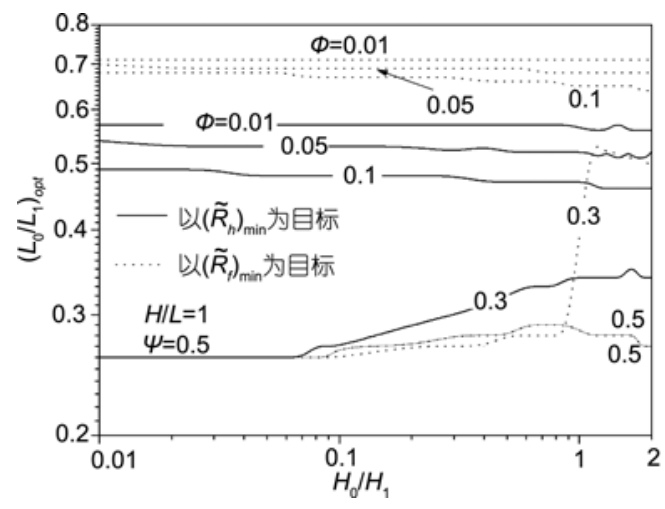

图 $3 \Phi$ 对 $\left(L_{0} / L_{1}\right)_{o p t}-H_{0} / H_{1}$ 关系的影响

当以 $\left(\widetilde{R_{h}}\right)_{\min }$ 为目标时, 由图 3 中实线可以看出, 当 $\Phi<0.1$ 时, 随着 $\Phi$ 增大, 对应给定的 $H_{0} / H_{1}$, $\left(L_{0} / L_{1}\right)_{o p t}$ 依序减小; 对于给定的 $\Phi$ 值, $\left(L_{0} / L_{1}\right)_{o p t}$ 随 $H_{0} / H_{1}$ 增大而基本保持不变. 当 $\Phi>0.1$, $H_{0} / H_{1}>0.07$ 时, $\left(L_{0} / L_{1}\right)_{\text {opt }}$ 仍然随 $\Phi$ 增大而减小, 但 $H_{0} / H_{1}<0.07$ 时, $\left(L_{0} / L_{1}\right)_{\text {opt }}$ 限于约束条件都只能 取到最小值 0.26 而不能继续减小; 当 $\Phi=0.3$, $0.07<H_{0} / H_{1}<2$ 时, $\left(L_{0} / L_{1}\right)_{\text {opt }}$ 随 $H_{0} / H_{1}$ 的增大总 体上呈增长趋势; 当 $\Phi=0.5,0.09<H_{0} / H_{1}<2$ 时, $\left(L_{0} / L_{1}\right)_{\text {opt }}$ 随 $H_{0} / H_{1}$ 的增大先增长而后稍降低.

比较图 3 中虚线和实线可以看出, 当 $\Phi \leqslant 0.1$ 时, 对应 $\left(\widetilde{R_{t}}\right)_{\min }$ 的 $\left(L_{0} / L_{1}\right)_{\text {opt }}$ 比对应 $\left(\widetilde{R_{h}}\right)_{\min }$ 的 $\left(L_{0} / L_{1}\right)_{\text {opt }}$ 要大; 随着 $\Phi$ 增大, 对应给定的 $H_{0} / H_{1},\left(L_{0} / L_{1}\right)_{\text {opt }}$ 仍然依序减小; 对于给定的 $\Phi$ 值, $\left(L_{0} / L_{1}\right)_{o p t}$ 随 $H_{0} / H_{1}$ 增大而基本保持不变. 但当 $\Phi=0.3$ 时, $\left(L_{0} / L_{1}\right)_{\text {opt }}$ 出现了一个较大的阶跃.

图 4 给出了 $\Phi$ 对 $\left(\widetilde{R_{h}}\right)_{\min }-H_{0} / H_{1}$ 关系的影响. 计 算表明, 对于给定的 $\Phi,\left(\widetilde{R_{h}}\right)_{\text {min }}$ 随 $H_{0} / H_{1}$ 增大呈现
增长趋势, 不存在最优的 $H_{0} / H_{1}$, 并且随着 $\Phi$ 增大 $\left(\widetilde{R_{h}}\right)_{\min }$ 的增长幅度是增大的. 当 $H_{0} / H_{1}<0.05$ 时, $\Phi=0.5$ 所对应的 $\left(\widetilde{R_{h}}\right)_{\min }$ 是最小的, 系统的传热性能 最好. 但是, 当 $H_{0} / H_{1}>1$ 时, 随着 $\Phi$ 增大, 对应给 定的 $H_{0} / H_{1},\left(\widetilde{R_{h}}\right)_{\min }$ 反而增大. 这表明, 由于 $\mathrm{T}$ 形腔 所占矩形域体积的限定, 虽然增大了冷却空腔的体 积, 但其冷却效能却在尺寸约束下受到极大的限制, 沵耗散增加, 传热性能降低.

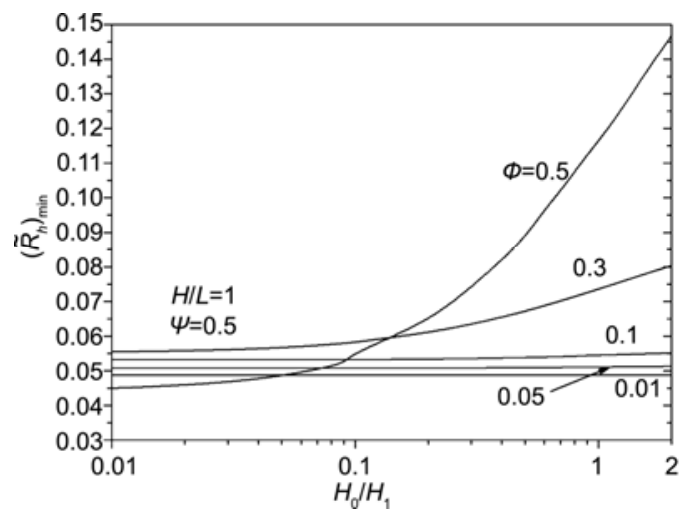

图 $4 \Phi$ 对 $\left(\widetilde{R_{h}}\right)_{\min }-H_{0} / H_{1}$ 关系的影响

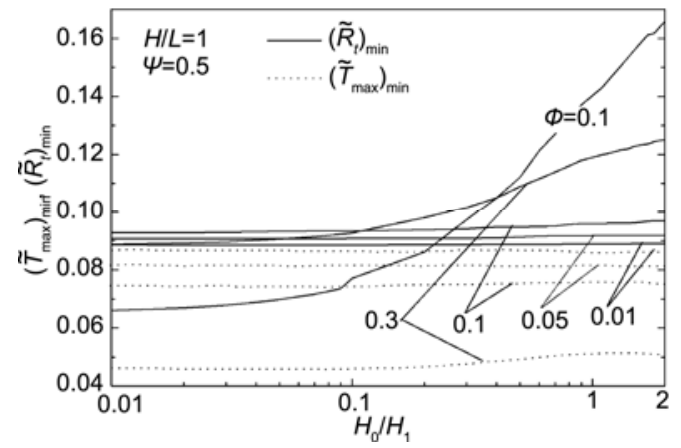

图 $5 \Phi$ 对 $\left(\widetilde{R}_{t}\right)_{\min }-H_{0} / H_{1}$ 关系和 $\widetilde{T}_{\text {max }}-H_{0} / H_{1}$ 关系的影响

图 5 给出了 $\Phi$ 对 $\left(\widetilde{R_{t}}\right)_{\min }-H_{0} / H_{1}$ 关系(实线)和 $\widetilde{T}_{\text {max }}-H_{0} / H_{1}$ 关系(虚线, 文献[36]的优化结果)的影 响. 实线表明, 优化结果与以 $\left(\widetilde{R_{h}}\right)_{\min }$ 为目标的优化 结果具有一定的相似性, 即对于给定的 $\Phi,\left(\widetilde{R_{h}}\right)_{\min }$ 随 $H_{0} / H_{1}$ 增大呈现增长趋势, 不存在最优的 $H_{0} / H_{1}$, 并且随着 $\Phi$ 增大 $\left(\widetilde{R_{h}}\right)_{\min }$ 增长的幅度是增大的. 当 $H_{0} / H_{1}<1.1$ 时, $\Phi=0.5$ 所对应的 $\left(\widetilde{R_{t}}\right)_{\text {min }}$ 是最小的, 系统的最大热阻最小. 但是, 当 $H_{0} / H_{1}>1.3$ 时, 对应 给定的 $H_{0} / H_{1},\left(\widetilde{R_{t}}\right)_{\min }$ 随着 $\Phi$ 增大反而增大. 这与 虚线所示规律有很大不同。虚线表明，在 $0.01<H_{0} / H_{1}<2$ 的整个计算区间, $\left(\widetilde{T}_{\max }\right)_{\text {min }}$ 随着 $\Phi$ 
增大而降低. 造成这种差异的原因在于, 增大 $\Phi$ 对 于降低系统中最高温度是有效的, 但由于增大 $\Phi$ 的 同时减小了产热区域，也就减小了系统的散热负荷， 而 $\left(\widetilde{R_{t}}\right)_{\min }$ 是一个相对兼顾了上限温度和散热负荷的 指标, 所以 $\Phi$ 对 $\left(\widetilde{R_{t}}\right)_{\text {min }}-H_{0} / H_{1}$ 关系的影响就比 $\Phi$ 对 $\widetilde{T}_{\text {max }}-H_{0} / H_{1}$ 关系的影响更为复杂.

$2.2 \mathrm{~T}$ 形腔所占矩形域占比 $\psi$ 对系统构形及其性能 的影响

取 $\Phi=0.1$, 研究 T 形腔所占矩形域占比 $\psi$ 对最优 系统构形及其性能的影响. 图 6 给出了以 $\left(\widetilde{R_{h}}\right)_{\min }$ 为 目标时 $\psi$ 对 $\left(L_{0} / L_{1}\right)_{\text {opt }}-H_{0} / H_{1}$ 关系的影响, 图 7 给出 了对应图 6 中系统构形的 $\left(\widetilde{R_{h}}\right)_{\min }$; 图 8 给出了以 $\left(\widetilde{R_{t}}\right)_{\min }$ 为目标时 $\psi$ 对 $\left(L_{0} / L_{1}\right)_{o p t}-H_{0} / H_{1}$ 关系的影响, 图 9 给出了 $\psi$ 对 $\left(L_{0} / L_{1}\right)_{o p t}-H_{0} / H_{1}$ 关系和 $\widetilde{T}_{\text {max }}-H_{0} / H_{1}$ 关系的影响.

由图 6 可知, 在整个计算区间, 对应给定的 $H_{0} / H_{1}$, $\left(L_{0} / L_{1}\right)_{\text {opt }}$ 随着 $\psi$ 增大而增长; 当 $\psi$ 给定时, $\left(L_{0} / L_{1}\right)_{\text {opt }}$

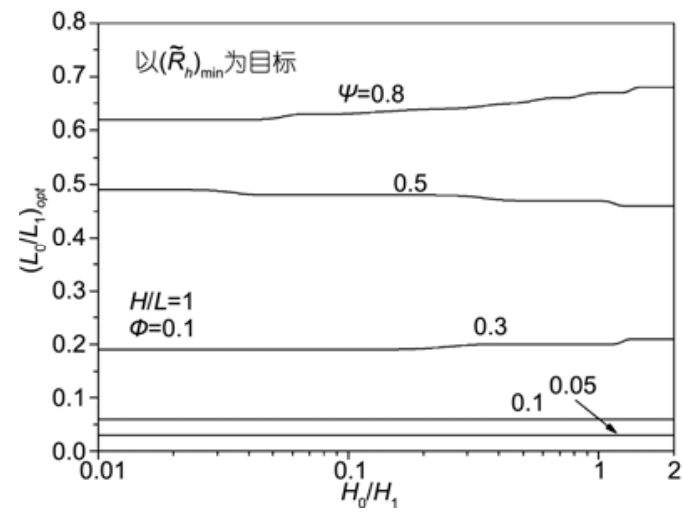

图 $6 \psi$ 对 $\left(L_{0} / L_{1}\right)_{o p t}-H_{0} / H_{1}$ 关系的影响

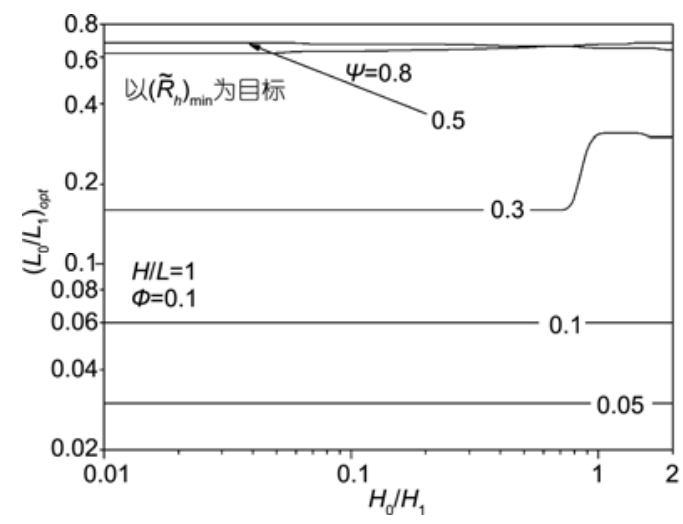

图 $8 \psi$ 对 $\left(L_{0} / L_{1}\right)_{o p t}-H_{0} / H_{1}$ 关系的影响
对 $H_{0} / H_{1}$ 的变化并不敏感, 变化幅度小于 $9 \%$. 由图 8 可知, 对于 $\psi<0.5$ 的情况, 对应给定的 $H_{0} / H_{1}$, $\left(L_{0} / L_{1}\right)_{\text {opt }}$ 仍然随着 $\psi$ 增大而增长; 但是当 $\psi=0.3$ 时, $\left(L_{0} / L_{1}\right)_{o p t}-H_{0} / H_{1}$ 曲线出现了一个阶跃.

比较图 6 和 8 的结果可知, 当 $\psi=0.3,0.5$ 时, 分 别以 $\left(\widetilde{R_{h}}\right)_{\min }$ 和 $\left(\widetilde{R_{t}}\right)_{\min }$ 为目标所得的最优构形明显不 同. 当 $\psi=0.3 、 H_{0} / H_{1}<0.9$ 时, 以 $\left(\widetilde{R_{h}}\right)_{\min }$ 为目标所 得的 $\left(L_{0} / L_{1}\right)_{o p t}$ 更大一些, 当 $H_{0} / H_{1}>0.9$ 时, 由于以 $\left(\widetilde{R_{t}}\right)_{\text {min }}$ 为目标所得的 $\left(L_{0} / L_{1}\right)_{\text {opt }}$ 出现阶跃, 所以比以 $\left(\widetilde{R_{h}}\right)_{\min }$ 为目标所得的 $\left(L_{0} / L_{1}\right)_{\text {opt }}$ 更大一些. 当 $\psi=0.5$ 时, 以 $\left(\widetilde{R_{h}}\right)_{\min }$ 为目标所得的 $\left(L_{0} / L_{1}\right)_{\text {opt }}$ 始终要小一些. 由于尺寸的限制, 当 $\psi=0.8$ 时, 对应各 $H_{0} / H_{1}$ 的 $\left(L_{0} / L_{1}\right)_{o p t}$ 只能取得 $L_{0} / L_{1}$ 的最大值; 当 $\psi=0.05,0.1$ 时, 对应各 $H_{0} / H_{1}$ 的 $\left(L_{0} / L_{1}\right)_{\text {opt }}$ 均取得 $L_{0} / L_{1}$ 的最小 值, 导致分别以 $\left(\widetilde{R_{h}}\right)_{\text {min }}$ 和 $\left(\widetilde{R_{t}}\right)_{\text {min }}$ 为目标所得的最优 构形无差异.

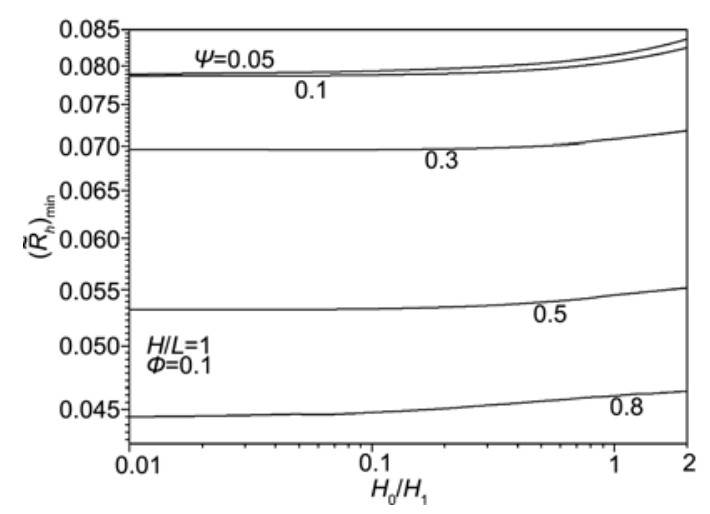

图 7 对应图 6 中系统构形的 $\left(\widetilde{R_{h}}\right)_{\min }$

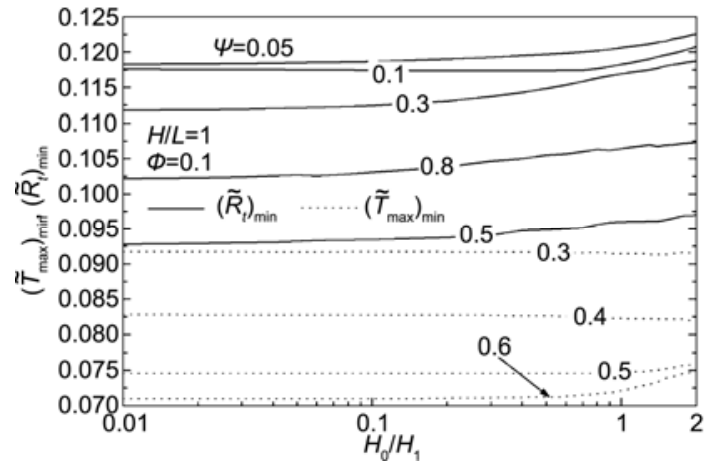

图 $9 \psi$ 对 $\left(\widetilde{R}_{t}\right)_{\min }-H_{0} / H_{1}$ 关系和 $\left(\widetilde{T}_{\text {max }}\right)_{\min }-H_{0} / H_{1}$ 关系的影响 
由图 7 可知, 对应给定的 $H_{0} / H_{1},\left(\widetilde{R_{h}}\right)_{\text {min }}$ 随 $\psi$ 增大而减小. 这说明, 当冷却空腔体积一定的时候, 通过在给定区域合理选用 $\mathrm{T}$ 形腔所占矩形域大小(也 就是说, $\mathrm{T}$ 形腔具有更大的自由度)可以更加充分地发 挥空腔的冷却效能, 提高系统传热性能. 当 $\psi$ 给定时, $\left(\widetilde{R_{h}}\right)_{\min }$ 随 $H_{0} / H_{1}$ 增大而缓慢增长.

由图 9 可知, 对于 $\psi<0.5$ 的情况, 对应给定的 $H_{0} / H_{1},\left(\widetilde{R_{t}}\right)_{\min }$ 随 $\psi$ 增大而减小. 文献[36]的研究结 果(虚线)表明, 当 $\psi$ 从 0.3 增大到 0.6 时, $\left(\widetilde{T}_{\text {max }}\right)_{\text {min }}$ 也 随 $\psi$ 增大而减小。但是当 $\psi=0.8$ 时, 对应给定的 $H_{0} / H_{1},\left(\widetilde{R_{t}}\right)_{\min }$ 反而增大了. 这说明, 当冷却空腔体 积一定的时候, 在一定范围内可以通过在给定区域 合理设计 $\mathrm{T}$ 形腔所占矩形域大小, 更加充分地发挥空 腔的冷却效能, 有效降低系统最大热阻; 当 $\psi=0.8$ 时, 由于尺寸约束而使得最大热阻最小化的优化设计受 到实际限制. 因此, 在工程设计中, $\psi$ 和 $\Phi$ 是两个需 要结合实际情况进行合理匹配的参数, 既要合理选择 空腔体积又要有效地设计 $\mathrm{T}$ 形腔所占矩形域大小, 充 分发挥空腔的冷却效能, 最大限度地提高系统整体性 能. 当 $\psi$ 给定时, $\left(\widetilde{R}_{t}\right)_{\min }$ 随 $H_{0} / H_{1}$ 增大而增长.

\section{3 释放 $\mathrm{T}$ 形腔所占矩形域占比 $\psi$ 的优化分析}

图 10 给出了进一步释放 $\mathrm{T}$ 形腔所占矩形域占比 $\psi$, 以 $L_{0} / L_{1}, H_{0} / H_{1}$ 和 $H_{1} / L_{1}$ 为自由度的三维优化 结果. 计算表明, 低 $H_{1} / L_{1}$ 数时, $\left(\widetilde{R_{h}}\right)_{\text {min }}$ 和 $\left(\widetilde{R_{t}}\right)_{\text {min }}$ 均 随 $H_{1} / L_{1}$ 的增长缓慢减小, 两种优化目标所对应的 $\left(H_{0} / H_{1}\right)_{0}$ 均大幅降低, 且分别当 $H_{1} / L_{1}=0.170,0.256$ 时都降低至计算的下限值 0.01 ; 但 $\left(L_{0} / L_{1}\right)_{o o}$ 相对稳

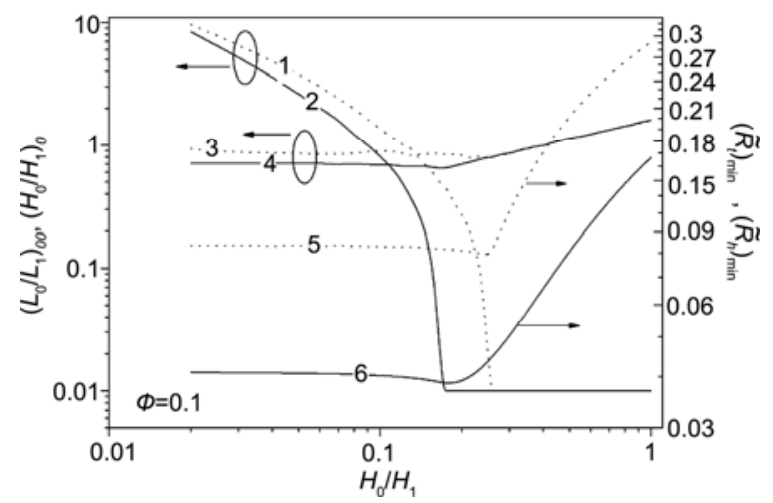

图 103 自由度优化结果

以 $\left(\widetilde{R_{h}}\right)_{\min }$ 为目标; -...- 以 $\left(\widetilde{R_{t}}\right)_{\min }$ 为目标 $1,2-\left(H_{0} / H_{1}\right)_{0} ; 3,4-\left(L_{0} / L_{1}\right)_{00} ; 5-\left(\widetilde{R_{t}}\right)_{\min } ; 6-\left(\widetilde{R_{h}}\right)_{\min }$
定, 当 $H_{1} / L_{1} \geqslant 0.246$ 时, 两种不同目标的优化具有相 同的 $\left(L_{0} / L_{1}\right)_{o o}$. 当 $H_{1} / L_{1}=0.176,0.246$ 时, $\left(\widetilde{R_{h}}\right)_{m m}$ 和 $\left(\widetilde{R_{t}}\right)_{m m}$ 分别取得最小值 0.038601 和 0.079474 .3 .2 节 的优化中, $\left(\widetilde{R_{h}}\right)_{m m}$ 和 $\left(\widetilde{R_{t}}\right)_{m m}$ 的最小值分别为 0.044456 和 0.092816 , 可见通过释放 T 形腔所占矩形 域占比 $\psi$ 的约束, 给予空腔更大自由度, 使得 $\left(\widetilde{R_{h}}\right)_{m m}$ 和 $\left(\widetilde{R_{t}}\right)_{m m}$ 的降幅分别达到了 $13.17 \%$ 和 $14.37 \%$, 系统 传热性能更佳. 高 $H_{1} / L_{1}$ 数时, $\left(\widetilde{R_{h}}\right)_{m m}$ 随着 $H_{1} / L_{1}$ 的 增大接近对数增长, $\left(H_{0} / H_{1}\right)_{0}$ 值没有出现反弹, $\left(L_{0} / L_{1}\right)_{o o}$ 值则持续增长.

对图 10 所示数值结果采用最小二乘法拟合函数, 分别得到如下关联关系式

$\left(\widetilde{R_{h}}\right)_{m m}=0.08641\left(\frac{H_{1}}{L_{1}}\right)^{0.10323}\left(\frac{H_{0}}{H_{1}}\right)_{o}^{0.03757}\left(\frac{L_{0}}{L_{1}}\right)_{o o}^{1.34922}$

$\left(\widetilde{R_{t}}\right)_{m m}=0.10182\left(\frac{H_{1}}{L_{1}}\right)^{-0.02990}\left(\frac{H_{0}}{H_{1}}\right)_{o}^{-0.05231}\left(\frac{L_{0}}{L_{1}}\right)_{o o}^{1.72103}$.

最大拟合相对误差分别 $12.42 \%$ 和 $8.96 \%$.

\section{4 优化效果的对比分析}

图 11 和 12 分别给出了 $\Phi$ 和 $\psi$ 对 2.1, 2.2 节中最优 构形所对应的 $\Delta \widetilde{\bar{T}}-H_{0} / H_{1}$ 关系的影响. 计算结果表 明, 当 $\Phi=0.3 、 H_{0} / H_{1}<1.1$ 时, 限于尺寸约束条件, 分别以 $\left(\widetilde{R_{t}}\right)_{\min }$ 和 $\left(\widetilde{R_{h}}\right)_{\min }$ 为指标所得的构形相同或相 近, 从而使得对应的系统传热平均温差也相同或相近; 当 $H_{0} / H_{1}>1.1$ 时, 以 $\left(\widetilde{R_{h}}\right)_{\text {min }}$ 为目标的优化明显地降 低了系统传热平均温差. 比较两图中其余实线和虚线 均可以看出, 在约束条件相同的情况下, 以 $\left(\widetilde{R_{h}}\right)_{\text {min }}$ 为 目标的优化比以 $\left(\widetilde{R_{t}}\right)_{\text {min }}$ 为目标的优化有效地降低了 传热平均温差, 改善了系统传热性能. 由式(15)

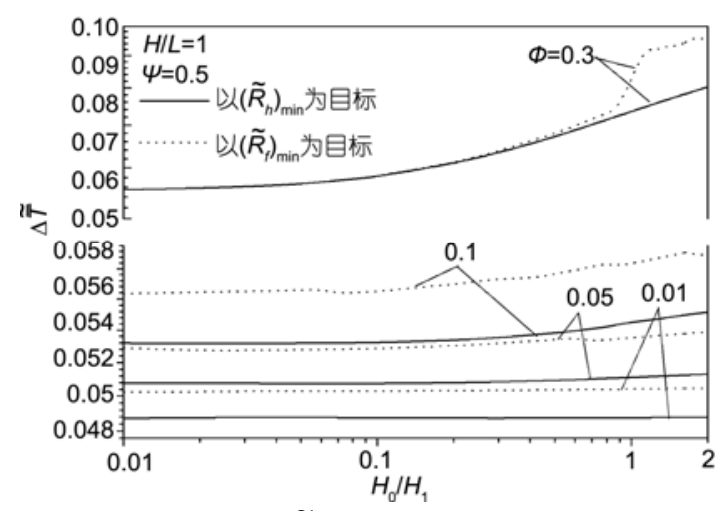

图 $11 \Phi$ 对 $\Delta \widetilde{\bar{T}}-H_{0} / H_{1}$ 关系的影响 


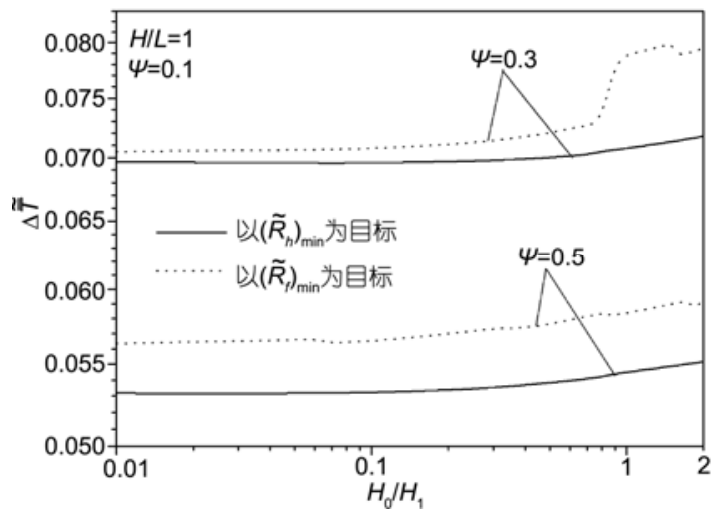

图 $12 \psi$ 对 $\Delta \widetilde{\bar{T}}-H_{0} / H_{1}$ 关系的影响

可知, 传热平均温差的变化规律与 $2.1,2.2$ 节中 $\left(\widetilde{R_{h}}\right)_{\min }$ 的变化规律一致.

图 3 和 8 表明, 当 $\psi=0.5, \Phi=0.3$ 和 $\psi=0.3, \Phi=$ 0.1 时, 以 $\widetilde{R}_{t}$ 最小化为目标的优化所得的 $\left(L_{0} / L_{1}\right)_{\text {opt }}$ 都 出现了阶跃, 这充分反映出以刻画系统极限特征的 目标函数优化系统构形具有不连续性, 是其片面性 和局限性的一种表现. 以 $\widetilde{R}_{t}$ 最小化进行优化主要是 出于极限安全考虑; 而以 $\widetilde{R}_{h}$ 最小化进行优化, 目的 是降低系统的平均传热温差, 提高系统整体的传热 性能. 因此, 工程上对相关问题进行热优化设计时, 在兼顾上限温度(最大热阻)的情况下应该尽量选择 对应当量热阻较小的构形设计.

\section{3 结论}

炇耗散极值原理给出了新的传热优化的理论依 据和评判标准. 本文针对现代工程领域中普遍存在 的导热固体中包含开口空腔的传热模型, 在引入积 耗散无量纲当量热阻的基础上, 以其最小化为目标 对方形固体中的 $\mathrm{T}$ 形开口空腔进行了构形优化, 结果 表明基于积耗散极值原理可以设计出系统传热性能
最优的几何结构. 修正了文献[36]中无量纲总(最大) 热阻的表达式, 并采用同样的数值方法进行了优化 和分析. 数值结果表明, 分别对应本文两种热阻指标 的最优系统结构差别很大. 当 $\psi=0.5, \Phi<0.1$ 时, 对 应 $\left(\widetilde{R_{t}}\right)_{\min }$ 的 $\left(L_{0} / L_{1}\right)_{o p t}$ 比对应 $\left(\widetilde{R_{h}}\right)_{\min }$ 的 $\left(L_{0} / L_{1}\right)_{o p t}$ 要大; 当 $\psi=0.3, \Phi=0.1, H_{0} / H_{1}<0.9$ 时, 以 $\left(\widetilde{R_{h}}\right)_{\text {min }}$ 为目 标所得的 $\left(L_{0} / L_{1}\right)_{o p t}$ 更大一些, 当 $H_{0} / H_{1}>0.9$ 时, 由 于以 $\left(\widetilde{R_{t}}\right)_{\min }$ 为目标所得的 $\left(L_{0} / L_{1}\right)_{\text {opt }}$ 出现阶跃, 所以比 以 $\left(\widetilde{R_{h}}\right)_{\min }$ 为目标所得的 $\left(L_{0} / L_{1}\right)_{o p t}$ 更大一些. 当 $\psi=0.5, \Phi=0.1$ 时, 以 $\left(\widetilde{R}_{h}\right)_{\text {min }}$ 为目标所得的 $\left(L_{0} / L_{1}\right)_{\text {opt }}$ 始终要小一些.

无量纲最大热阻 $\left(\widetilde{R_{t}}\right)$ 是以局部极限特征刻画系 统整体性能, 以其最小化为目标优化系统构形有一 定的片面性和局限性, 出现了不连续性. 基于䛈耗散 定义的当量热阻能够反映多维传热系统整体的平均 散热性能, 工程上对相关问题进行热优化设计时, 在 兼顾上限温度(最大热阻)的情况下应该尽量选择对 应当量热阻较小的构形设计. 当 $\mathrm{T}$ 形腔所占矩形域体 积受限时, 增大冷却空腔体积并不一定能够有效减 小当量热阻和最大热阻; 当给定冷却空腔体积时, 在 一定范围内可以通过合理设计 $\mathrm{T}$ 形腔所占矩形域大小 有效降低当量热阻和最大热阻. 因此, 在工程设计 中, $\psi$ 和 $\Phi$ 是两个需要结合实际情况进行合理匹配的 参数, 既要合理选择空腔体积又要有效地设计 $\mathrm{T}$ 形腔 所占矩形域大小, 充分发挥空腔的冷却效能, 最大限 度地提高系统整体性能. 空腔自由度越大, 系统性能 更佳. 通过函数拟合, 分别得到了当量热阻和最大热 阻与 3 自由度几何参数的优化关联式, 对实际工程设 计具有一定指导意义.

\section{参考文献}

1 Guo Z Y, Li D Y, Wang B X. A novel concept for convective heat transfer enhancement. Int J Heat Mass Transfer, 1998, 41: $2221-2225[\mathrm{DOI}]$

2 过增元. 对流换热的物理机制及其控制：速度场与热流场的协同. 科学通报, 2000, 45: 2118- 2122

3 过增元, 魏澍, 程新广. 换热器强化的场协同原理. 科学通报, 2003, 48: 2324-2327

4 Bejan A. Entropy Generation Minimization. New York: Wiley, 1996

5 Bejan A. Entropy generation minimization: the new thermodynamics of finite-size devices and finite-time processes. J Appl Phys, 1996, 
79: $1191-1218 \underline{\text { DOi] }}$

6 Chen L, Wu C, Sun F. Finite time thermodynamic optimization or entropy generation minimization of energy systems. J Non-Equibri Thermodyn, 1999, 24: 327-359

7 Bejan A. Constructal-theory network of conducting paths for cooling a heat generating volume. Trans ASME J Heat Transfer, 1997, 40: $799-816$

9 Bejan A, Rocha L A O, Lorente S. Thermodynamic optimization of geometry: T and Y-shaped constructs of fluid streams. Int J Thermal Sci, 2000, 39: 949-960[DOI]

10 Bejan A, Lorente S. Design with Constructal Theory. New Jersey: Wiley, 2008

11 Wang A H, Liang X G, Ren J X. Constructal enhancement of heat conduction with phase change. Int J Thermophys, 2006, 27: $126-138 \underline{\mathrm{DOI}]}$

$12 \mathrm{Yu} \mathrm{B,} \mathrm{Li} \mathrm{B.} \mathrm{Fractal-like} \mathrm{tree} \mathrm{networks} \mathrm{reducing} \mathrm{the} \mathrm{thermal} \mathrm{conductivity.} \mathrm{Phys} \mathrm{Rev} \mathrm{E,} \mathrm{2006,} \mathrm{73:} \mathrm{066302[DOI]}$

Wu W, Chen L, Sun F. On the "area to point” flow problem based on constructal theory. Energy Convers Mgmt, 2007, 48: 101—105[DOI]

Zhou S, Chen L, Sun F. Optimization of constructal volume-point conduction with variable cross-section conducting path. Energy Convers Mgmt, 2007, 48: 106-111[DOI]

15 Xu X, Liang X, Ren J. Optimization of heat conduction using combinatorial optimization algorithms. Int J Heat Mass Transfer, 2007, 50: $1675-1682[\mathrm{DOI}]$

伍文君，陈林根，孙丰瑞. 导热优化的“树网”构造法的改进. 中国科学 E 辑：技术科学, 2006, 36: 773-781

Shah R K, Skiepko T. Entropy generation extrema and their relationship with heat exchanger effectiveness-Number of transfer unit behavior for complex flow arrangements. Trans ASME J Heat Transfer, 2004, 126: 994-1002[DOI]

Guo Z Y, Zhu H Y, Liang X G. Entransy-A physical quantity describing heat transfer ability. Int J Heat Mass Transfer, 2007, 50: $2545-2556[\mathrm{DOI}]$

过增元. 热学中的新物理量. 工程热物理学报, 2008, 29: 112-114

过增元, 程新广, 夏再忠. 最小热量传递势容耗散函数原理及其在导热优化中的应用. 科学通报, 2003, 48: 21一25

程新广，李志信，过增元. 基于仿生优化的高效导热通道的构造. 中国科学 E 辑: 技术科学, 2003, 33: 251-256

韩光泽, 过增元. 不同目的热优化目标函数：热量传递势容损耗与熵产. 工程热物理学报, 2007, 27: 811一813

程新广, 孟继安, 过增元. 导热优化中的最小传递势容耗散与最小摘产. 工程热物理学报, 2005, 26: 1036

韩光泽, 过增元. 导热能力损耗的机理及其数学表述. 中国电机工程学报, 2007, 27: 98-102

朱宏华, 陈泽敬, 过增元. 秌耗散极值原理的电热模拟实验研究. 自然科学进展, 2007, 17: 1692-1698

柳雄斌, 过增元, 孟继安. 换热器中的䍉耗散与热阻分析. 自然科学进展, 2008, 18: 1186-1190

宋伟明, 孟继安, 梁新刚, 等. 一维换热器中温差场均匀性原则的证明. 化工学报, 2008, 59: 2460-2464

柳雄斌, 孟继安, 过增元. 换热器参数优化中的摘产极值和炽耗散极值. 科学通报, 2008, 53: 3026-3029

吴晶, 梁新刚. 沵耗散极值原理在辐射换热优化中的应用. 中国科学 E 辑: 技术科学, 2009, 39: 272-277

魏曙㝘, 陈林根, 孙丰瑞. 基于矩形单元体的以积耗散最小为目标的“体点”导热构形优化.中国科学 E 辑：技术科学，2009, 39: $278-285$

魏曙哀, 陈林根, 孙丰瑞. 以秌耗散最小为目标的电磁体构形优化. 中国科学 E 辑: 技术科学, 2009, 待发表 